\title{
L'HORROR ALLA UNIVERSAL: TRASFORMARE I LIMITI IN UNA RISORSA
}

\author{
Mattia Guzzi ${ }^{1}$ \\ ${ }^{1}$ Affiliation not available
}

October 25, 2021

\section{Introduzione}

Questo elaborato si pone l'obbiettivo di descrivere e analizzare le modalità produttive del genere horror di casa Universal, nel cosiddetto periodo classico di Hollywood.

Dietro al successo di un genere, nell'era dello studio system 11Brunetta, Gian Piero, ed. Storia del cinema mondiale . Vol. 2. Einaudi (IT), 1999, non esiste solo un importante componente estetica di carattere prettamente filmico, cinematografico, ma anche, e soprattutto, un insieme di decisioni produttive, un programma distributivo, contratti col personale artistico, un casting e una scelta dei collaboratori, una previsione dei gusti del pubblico, amministrazione delle riprese e del budget, la compra vendita di licenze, accordi con gli esercenti ecc...

Tutto questo e molto altro si nasconde dietro ad un film, o ad un genere, prodotto da questa o quella casa di produzione in un periodo in cui, ad Hollywood, il cinema era, sì un evento culturale, ma anche e soprattutto una grande azienda profittevole.

Il cinema americano è un'arte classica, ma perché non ammirare in essa ciò che è più ammirevole? Cioè non solo il talento di questo o quel regista, ma anche e soprattutto il genio del sistema 22Schatz, Thomas. The genius of the system: Hollywood filmmaking in the studio era . Macmillan, 1996 (Bazin, 1958)

L'elaborato passerà in rassegna un po' di storia della casa Universal, per capirne le radici ideologiche, fino ad arrivare all'età del sonoro, così da concentrarsi sullo sviluppo e successo dell'horror nella casa, e descrivere come e perché ci si dedicò a questa tipologia di film.

\section{Carl Laemmle e la Universal}

Come William Fox, Adolph Zukor, e altri magnati del cinema americano, anche Carl Laemmle iniziò dal business dei nickelodeon in una grande città industriale come Chicago. Quando il potere del trust(M.C.C.P.) stava diminuendo e gli indipendenti piano piano si insinuavano nel nuovo regime, la Universal iniziava a diventare sempre più grande e Laemmle vendette tutti i teatri per concentrarsi esclusivamente nella produzione e distribuzione. Nel 1915 a Los Angeles Universal city era finita, e poteva rendere ormai 250 prodotti tra cortometraggi, cinegiornali, periodici e lungometraggi all'anno.

Carl Laemmle era un imprenditore, emigrato dalla Germania, ebreo, aveva il fiuto degli affari ma sapeva ben poco della produzione dei film. Fu una personalità importantissima per lo sviluppo di Hollywood, si pensi per esempio che fu lui a combattere in prima persona il trust di Edison, e che seppe per primo sfruttare il potente mezzo delle star per la fidelizzazione del pubblico e la minimizzazione del rischio produttivo11https://www.immigrantentrepreneurship.org/entries/carl-laemmle/. Mantenne sempre grossi contatti con il paese di provenienza e riuscì con le sue doti imprenditoriali a creare una catena di prodotti che sapevano adattarsi bene al mercato sia europeo che americano; questa internazionalità divenne poi il marchio distintivo della casa. 
Nel 1918 Irving Thalberg, un ragazzo visionario di appena ventidue anni, ricevette il controllo esclusivo di Universal City . Laemmle era da tempo affezionato e sicuro di quello che definiva "programma scientificamente equilibrato" di cortometraggi, cinegiornali, serie e servizi modesti; per lui la Universal era una fabbrica che doveva puntare alla quantità e alla qualità di prodotti visivi semplici e di spettro più ampio possibile per avere pubblico variegato e ampio22Schatz, Thomas. The genius of the system: Hollywood filmmaking in the studio era. Macmillan, 1996. Il rischio di questa pratica era molto basso e le entrate praticamente constanti e sicure. Nel 1920, sulla scia di questa pratica, la Universal aveva quasi cancellato del tutto il suo primo mercato.

Questa strategia produttiva, che mirava soprattutto a riempire gli schermi di provincia in zone rurali, o al limite delle periferie di alcune città, aveva avuto senso in passato, quando la concorrenza era bassa, ma negli anni Venti queste pratiche erano ormai obsolete e Thalberg se ne accorse. I centri urbani stavo sempre più riempiendosi demograficamente, la società stava cambiando di conseguenza e le altre case si stavano preparando a concentrarsi verticalmente, per avere più controllo sui propri prodotti. La Universal si rivolgeva ad un pubblico in constante contrazione, non aveva sale o teatri, e questo l'avrebbe potuta danneggiare a lungo andare. Thalberg aveva già intuito che era sui lungometraggi di prestigio e sulle star che bisognava puntare per rendere fruttuosi i guadagni, ma Laemmle non ne voleva sapere. Nel 1923 Thalberg passò alla MGM e la Universal perse una delle più grandi figure produttive della sua epoca.

Si è detto che Laemmle era emigrato dalla Germania, e di fatto l'Europa rappresentò per molti anni un buon mercato, ma anche un aspetto importante che diversificò i prodotti della Universal rispetto alle altre case di produzione. Avendo ancora forti legami col paese di provenienza, Laemmle negli anni raccolse un ampio campionario di collaboratori dalla Germania, il che contribuì a caratterizzare l'house style 33Brunetta, Gian Piero, ed. Storia del cinema mondiale . Vol. 2. Einaudi (IT), 1999 della casa che divenne la più "europea" d'America.

Uno dei più grandi registi della casa degli anni Venti fu infatti l'europeo Erich von Stroheim, che fece molti dei suoi capolavori con la Universal, dopo innumerevoli scontri con Thalberg che non apprezzava la sua poca attitudine alla programmazione. Infatti nonostante la Universal avesse suo grosso punto fermo nella produzione bassa ed esile di serie e cortometraggi, apprezzava anche, soprattutto nel periodo di Thalberg, opere di grandi registi, in modo molto più libero di come facevano altre case. La pressione produttiva di Thalberg alla fine infuse metodicità negli studi della Universal dove fino a quel momento non c'era mai stata.

Importante sottolineare come già dagli anni Venti la Universal iniziò a gettare le basi per quello che poi sarebbe diventato il proprio genere caratteristico nel decennio successivo. Infatti già nel 1922, con alla regia Stroheim uscì Femmine Folli (Foolish Wives ), uno dei primi horror americani con un elevato budget. Il film fu un successo in America che da sempre apprezzava le atmosfere europee dei film Universal e portò la casa a concentrare la produzione su questa tipologia di prodotti. Altri titoli horror degli anni Venti sono: Il gobbo di Notre Dame (The Hunchback of Notre Dame,Wallace Worsley, 1923), Il fantasma dell'Opera (The Phantom of the Opera,Rupert Julian, 1925), Il castello degli spettri (The Cat and the Canary, Paul Leni, 1927),L'uomo che ride (The Man Who Laughs, , 1928). Tutti film prestigiosi che coinvolgevano registi importanti, grandi cast, produzioni vistose e grandi investimenti. Nonostante quindi le basi fondamentali della Universal fossero le piccole produzioni, Laemmle puntava anche su poche opere di qualità per non permettere alla bassa produzione di sfregiare l'immagine della casa.

\section{Carl Laemmle Junior e l'horror}

Nel 1928 Carl Laemmle passò la direzione di Universal City alle mani del figlio, Carl Laemmle Junior, che aveva le stesse idee di Thalberg sul mercato di prima esecuzione. Da subito Junior Laemmle puntò sulla produzione di lungometraggi di alto livello, tagliando circa il 40\% di cortometraggi e serie, ottenendo un enorme successo conBrodway (Pál Fejös, 1929) e All'ovest niente di nuovo (All Quiet on the Western Front,Lewis Milestone, 1930). Il primo era un musical che già sfruttava la fortuna del neonato sonoro e il secondo un film drammatico di guerra che ricevette statuette all'Oscar. Junior Laemmle, dopo appena due anni di lavoro, stava portando la Universal lì dove Thalberg non era riuscito a fare. 
Il problema dell'epoca era che, come in altre case in quel periodo, arrivò il duro colpo della Depressione e la Universal dovette fare qualche passo indietro, dedicandosi all'intero palinsesto piuttosto che ai film di prestigio. Quindi il programma di Junior Laemmle di competere con le altre case con film prestigiosi, dovette per il momento rimanere sospeso, per attenuare la crisi che il settore stava percorrendo. La produzione di genere a basso costo quindi costrinse la Universal a operare con i propri mezzi e a sfruttare le proprie risorse, ed è proprio qui che si sono rivelati i punti di forza della casa.

In questo periodo la produzione della casa contava di formule cinematografiche standard come i film di gangster o i film di donne, ma il genere caratteristico degli anni 30' fu l'horror11Neale, Stephen. Genre and hollywood. Psychology Press, 2000. Il genere non nasce dal niente con il sonoro, ma si è visto che la Universal aveva già avuto alle spalle molti film dell'orrore, soprattutto per il fatto che ha sempre avuto a contratto molti tecnici europei, ma soprattutto tedeschi, che si portavano dietro molte delle lezioni espressioniste degli anni Venti. Ma fu con il sonoro che arrivò il maggior successo del genere.

In particolare fu con l'uscita di Dracula (Tod Browning, 1931) che la Universal comprese dove doveva puntare per ottenere il successo sperato. Il film è tratto dalla omonima pièce teatrale che aveva avuto successo a Broadway nel 1927, scritta da Hamilton Deane e John Baldeston. Junior Laemmle lo aveva immaginato come una produzione di prima esecuzione, perché si basava su una proprietà prevenduta e coinvolgeva talenti esterni come il regista Tod Browning della MGM e Bela Lugosi che aveva recitato nella versione teatrale. Il budget, in linea con la nuova operazione semplificata, fu molto ridotto per far fronte alla crisi, ma nonostante questo fu la produzione più ambiziosa del 1930. Era stato prodotto nella metà del tempo e con un quarto delle risorse di All Quiet on the Western Front e fu un enorme successo, tanto che ancora prima della sua riuscita sul mercato, Junior Laemmle aveva messo in porto il film successivo Frankenstein(James Whale, 1931)22https://horror-9.jbtarlofsky-filminstitute-h.com/.

Lo studio come si è detto coltivava il genere da diversi anni, e riempiva il buco dove le altre case non erano in grado di farlo. Carl Laemmle aveva costruito un forte sistema di distribuzione internazionale, soprattutto in Europa, mantenendo vitali le immagini dei suoi film con lo stile di registi europei che aveva nel corso degli anni reclutato all'estero. Molti di loro erano reclute tedesche che avevano una tradizione dell'orrore gotico, ma anche del cinema espressionista33Gomery, Douglas. The Hollywood studio system: A history . Bloomsbury Publishing, 2019. La casa quindi fin dall'inizio sapeva lavorare bene con le immagini inquietanti, oscure e da incubo, una caratteristica che era unica nel suo genere alla Universal e che permise una certa ecletticità distributiva. L'atmosfera scura e inquietante dei film horror, permetteva anche banalmente, di abbassare i costi dell'illuminazione e delle scenografie44Prince, Stephen, ed. The horror film . Rutgers University Press, 2004; in più il genere non aveva bisogno di grandi star, che la Universal in quegli anni non aveva, ma di attori particolari un po' insoliti, come Lon Chaley, l'unica vera "star" di quel periodo. Tutto questo concentrato di qualità economiche del genere contribuì al suo successo e permise alla Universal di restare in equilibrio finanziario nei primi anni della Depressione.

Il film successivo a Dracula, fu quindiFrankenstein 55http://www.ondacinema.it/film/recensione/frankensteinwhale.html, sempre tratto dalla versione per il teatro, e con il regista James Whale che gli donò un clima ancora più espressionista del Dracula di Browning. Trentacinque giorni di riprese, un budget da 300.000 dollari e un altro successo commerciale strepitoso, che confermò la validità nel 1931 della nuova strategia di mercato della Universal.

Karl Freud, direttore della fotografia, che aveva partecipato allo stile visivo di Dracula e Frankenstein, venne laureato alla regia con La mummia (The Mummy, 1933), che ottenne anche lei grande successo e assicurò a Freud, per parecchi anni, il posto nei ranghi dei registi della Universal.

Un altro significativo film del periodo fu Il castello maledetto (The Old Dark House, James Whale, 1932) interpretato da Boris Karloff e Bela Lugosi, le due star del genere; il film fondeva atmosfere horror e comicità, testimoniando la flessibilità del genere e aprendolo a nuove eventuali sfaccettature. La coppia di attori (Karloff-Lugosi) proseguì la strada con un altro film importante come The Black Cat (Edgar G. Ulmer , 1934), secondo adattamento di Poe. Dopo qualche film di esperienza la Universal aveva imparato a gestire i propri 
prodotti in maniera molto più economica, soprattutto perché si basava su unità di programmazione. The Black Cat costò 91.125 dollari, un prezzo davvero irrisorio e si rivelò il miglior successo del 34', testimoniando come l'horror in quel periodo interessava il pubblico su tutti i mercati.

In questi anni la Universal si era fatta un nome e un proprio stile, il personale era scandito in potenti unità di produzione che sapevano essere veloci, economici e di qualità. Da non dimenticare anche tutta la componente di trucco, scenografie, musica, e di costumi che andavano sempre di più a raffinarsi con la pratica. La casa vantava inoltre della coppia di attori Karloff-Lugosi amati dal pubblico e riconoscibili, di grandi registi del genere, primo fra tutti Whale, e anche grandi sceneggiatori che erano in grado di adattare al meglio romanzi classici, come John Baldeston. Un grosso team che stava cogliendo i suoi frutti e che funzionava come una gigantesca macchina per portare la Universal fuori dalla crisi66Nowell, Richard, ed. Merchants of Menace: The Business of Horror Cinema . Bloomsbury Publishing USA, 2014.

In un periodo in cui gli altri studi integrati stavano subendo grosse perdite, la Universal riuscì con la formula dell'horror a restare a galla consolidando la sua posizione di minor, con una solida struttura di produzione e distribuzione, ma senza il peso di una catena di teatri, soluzione che nel periodo della depressione aveva più senso che mai.

\section{La caduta dei Laemmle}

Durante gli anni più grossi della depressione, 1931-33, quindi la Universal riuscì con i suoi mezzi a mantenere basse le perdite, producendo modesti film di genere, periodici e cortometraggi. Il film horror è stato cruciale in questo e ha permesso alla casa di stabilire e consolidare il suo genere specifico. Nel 1934 quindi sembrava che Junior Laemmle avesse acquisito le capacità imprenditoriali e di leadership, per essere in grado ormai di tracciare la rinascita dello studio. Così ritornò al progetto di fine anni Venti, per un ampiamento di film di prestigio che la Depressione aveva bloccato. Allargò quindi la produzione di horror e cercò di creare lo spazio per altri generi con la stessa strategia che aveva usato per i film dell'orrore. Un successo del periodo fu La moglie di Frankenstein (Bride of Frankenstein, James Whale, 1935) girato con un budget da film di seria A, che univa genere horror e commedia nera. Purtroppo però fu l'ultimo film dell'orrore girato dal miglior regista del genere della Universal. Whale voleva mettere le mani su generi più prestigiosi, così sulla scia di quella nuova strategia di competizione verso le produzioni di prestigio, Junior Laemmle gli assegnò un melodramma di alto livello. Assieme a Al di là delle tenebre (Magnificent Obsession,John M. Stahl, 1935), il film di Whale La canzone di Magnolia (Show Boat,1936) contribuì all'indebitamento della casa, che nel 1936 passò sotto le mani di J. Cheewer Codwin, che licenziò Junior Laemmle, l'uomo dell'orrore dalla Universal. Carl Laemmle venne per un primo momento mantenuto all'interno della casa, ma alla fine nel 1939 morì, e con lui in parte iniziò a cedere anche lo studio system 11Brunetta, Gian Piero, ed. Storia del cinema mondiale. Vol. 2. Einaudi (IT), 1999. Junior invece non trovò più un posto nella grande industria di Hollywood, ma i suoi frutti rimasero nella Universal anche dopo la improvvisa uscita. Si pensi che nel 2004, la Universal ha lanciato una serie di DVD che presentava le versioni rimasterizzate diDracula, Frankenstein , The Mummy, Il castello maledetto ecc....

Nel 1939 la Universal non era finalmente più in rosso e, trovandosi alle soglie dell'era d'oro dei B-movies e serials, si apriva davanti a lei un decennio radioso, data la propensione naturale della casa per queste tipologie di prodotto. Per un po' Rogers, il nuovo direttore di produzione, abbandonò le formule dell'horror per puntare su Deanna Durbin, un ex stella della MGM, e il suo genere caratteristico, il musical. Il genere ottenne grosso successo ma la grande mole di personaggi dell'orrore popolari degli anni 30' non poteva rimanere inutilizzata. Così, dopo un breve momento di stallo, Rogers rilanciò la produzione dell'orrore con spin-off e sequel, comeIl terrore di Frankenstein (The Ghost of Frankenstein,Erle C. Kenton, 1942), Il figlio di Dracula (Son of Dracula, Robert Siodmak, 1943), Al di là del mistero (The House of Frankenstein, Erle C. Kenton, 1944), La casa degli orrori (House of Dracula, Erle C. Kenton, 1945) ecc... Mentre le unità di produzione horror del decennio precedente si erano perse, l'identità del marchio che era stata attribuita da tempo alla società, attraverso la produzione orrorifica degli anni Trenta, persistette22Nowell, Richard, ed. Merchants of Menace: The Business of Horror Cinema. Bloomsbury Publishing USA, 2014. Così la Universal ripuntò su ciò che negli anni Trenta l'aveva resa famosa, ossia i mostri. Così l'horror di Junior 
Laemmle si era trasformato in un prodotto di serie B, della durata di massimo 70 minuti, con un programma di sequel, come per i film di Frankenstein, e in un sistema che puntava più sulla quantità che sulla qualità. Il cambio di gestione ebbe più lungimiranza e prudenza di Junior Laemmle e sfruttò al meglio il secondo mercato, capendo che la Universal non aveva i mezzi per puntare sulle produzioni di prestigio. Negli anni Quaranta alla fine si cambiò solo formula rispetto alla produzione orrorifica del decennio precedente, ma la sostanza rimaneva la stessa, ossia quella che era stata creata da Junior Laemmle, fu lui in fin dei conti il vero progettatore e "autore" del genere.

\section{Conclusione}

Come si è visto il successo dell'horror della Universal è frutto di diversi fattori, quali possono essere l'atmosfera europea della casa (grazie ad un grande numero di personale tedesco) o per la sua vocazione, non avendo mai voluto investire anche nell'esercizio, a ruolo di minor che ha permesso di investire lì dove altre case non lo facevano; ma anche, come si è visto, un po' per l'organizzazione in unità di produzione che permettevano alla casa di ricavare il massimo profitto dal minino dei mezzi. Tanti fattori che sicuramente convivono con altri, di cui non si è potuto parlare, per la esile natura di questo elaborato che purtroppo ha dei limiti molto stretti.

Negli anni Trenta il genere ha permesso alla casa di minimizzare le perdite dovute alla crisi dopo la Depressione, e ha saputo donare prestigio ad un genere che non era considerato molto dalle altre case di produzione in quegli anni. Con l'avvento del successo dei film di serie B, la Universal ha poi saputo ancora una volta sfruttare i propri limiti per farli diventare una risorsa, e il genere horror ha avuto un grosso successo anche negli anni Quaranta, spegnendosi poi nel decennio successivo perché i tempi erano cambiati e il pubblico aveva dimostrato interesse per altri generi, come la fantascienza.

Una grossa epopea di un genere all'interno di una casa di produzione che ancora ad oggi è rimasta in piedi, e la capacità del "genio del sistema" che ha saputo creare, sfruttare e far fruttare, un codice filmico quale quello dell'orrore. La storia di una casa di produzione e del lavoro di un grande uomo, che ha saputo investire e organizzare tutta la produzione di un genere: Carl Laemmle Junior.

\section{Bibliografia}

Brunetta, Gian Piero, ed. Storia del cinema mondiale . Vol. 2. Einaudi (IT), 1999

Gomery, Douglas. The Hollywood studio system: A history . Bloomsbury Publishing, 2019

Neale, Stephen. Genre and hollywood . Psychology Press, 2000

Nowell, Richard, ed. Merchants of Menace: The Business of Horror Cinema . Bloomsbury Publishing USA, 2014

Prince, Stephen, ed. The horror film . Rutgers University Press, 2004

Schatz, Thomas. The genius of the system: Hollywood filmmaking in the studio era. Macmillan, 1996

\section{Sitografia}

https://www.immigrantentrepreneurship.org/entries/carl-laemmle/

http://www.ondacinema.it/film/recensione/frankenstein-whale.html

https://horror-9.jbtarlofsky-filminstitute-h.com/ 